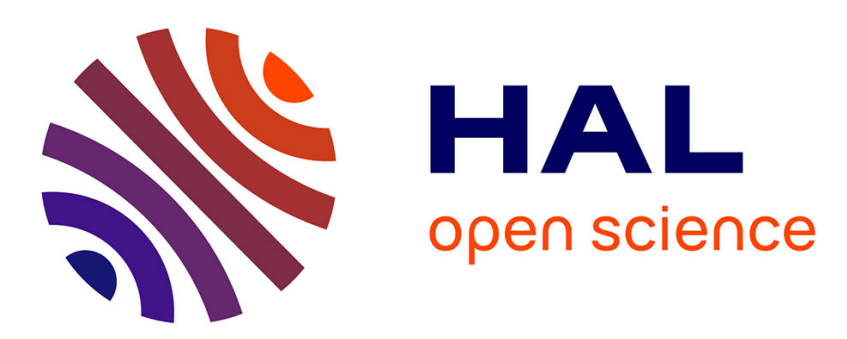

\title{
Bouc-Wen modeling and inverse multiplicative structure to compensate hysteresis nonlinearity in piezoelectric actuators.
}

Micky Rakotondrabe

\section{- To cite this version:}

Micky Rakotondrabe. Bouc-Wen modeling and inverse multiplicative structure to compensate hysteresis nonlinearity in piezoelectric actuators. IEEE Transactions on Automation Science and Engineering, 2011, 8 (2), pp.428-431. 10.1109/TASE.2010.2081979 . hal-00635669

\section{HAL Id: hal-00635669 \\ https://hal.science/hal-00635669}

Submitted on 25 Oct 2011

HAL is a multi-disciplinary open access archive for the deposit and dissemination of scientific research documents, whether they are published or not. The documents may come from teaching and research institutions in France or abroad, or from public or private research centers.
L'archive ouverte pluridisciplinaire HAL, est destinée au dépôt et à la diffusion de documents scientifiques de niveau recherche, publiés ou non, émanant des établissements d'enseignement et de recherche français ou étrangers, des laboratoires publics ou privés. 


\title{
Bouc-Wen modeling and inverse multiplicative structure to compensate hysteresis nonlinearity in piezoelectric actuators
}

\author{
Micky Rakotondrabe, member, IEEE
}

\begin{abstract}
A new approach to compensate the strong hysteresis nonlinearity in piezoelectric materials is proposed. Based on the inverse multiplicative scheme, the approach avoids models inversion as employed in existing works. The compensator is therefore simple to implement and does not require additional computation as soon as the direct model is available. The proposed compensation technique is valuable for hysteresis that are modeled with the Bouc-Wen set of equations.
\end{abstract}

Note to Practitioners - Since many years, the hysteresis nonlinearity in piezoelectric micro/nanoactuators (AFM, microgrippers, etc.) has been moving many researchers. Despite the high resolution and the high speed of these materials, the hysteresis strongly compromises the accuracy. If feedback control laws could easily improve the performances, their use in micro/nanoactuators and small systems are constrained by the luck of convenient sensors. On the one hand, accurate and high bandwidth sensors are very expensive and bulky (accelerometers, optical sensors). On the other hand, integrable sensors are highly sensitive to noises and are very fragile (strain gage). To surpass the use of sensors, feedforward control techniques have been used. These techniques represent a great interest in an integration/package point of view but the existing methods show a complexity in term of computation and implementation.

Three points have motivated the works presented in this article: 1) the need of high performances in micro/nanoactuation, 2) the shunning of the use of bulky sensors, 3) and the need of easily computable and implementable control techniques.

The content of the article focuses therefore on the design of a feedforward controller (compensator) with a special concern on the ease of computation and implementation. Of course, even if we present an application to a specific piezoelectric actuator, the proposed approach is also applicable to other systems whose the hysteresis is modeled with the Bouc-Wen expression.

Index Terms-Hysteresis compensation, Bouc-Wen model, inverse multiplicative scheme, piezoelectric actuators.

FEMTO-ST Institute, UMR CNRS 6174 - UFC / ENSMM / UTBM Automatic Control and Micro-Mechatronic Systems depart., AS2M

24, rue Alain Savary

25000 Besançon - France

mrakotone femto-st. fr

Paper type: short paper. First submission: March 2010.

\section{INTRODUCTION}

Piezoelectric materials, especially PZT ceramics (Lead Zirconate Titanate), are very prised in the development of micro/nanoactuators. This is due to the high resolution, the high bandwidth, the low cost and the simple treatment that piezoceramics offer. Unfortunately, piezoceramics exhibit strong hysteresis nonlinearity that undeniably compromises the final accuracy of the actuators and that may lead to unwanted harmonics.

Feedback control techniques seem to be the best way to reach overall substantial performances (accuracy, repeatability, disturbances and vibration rejection, uncertainty effect rejection, etc.) [1] [2]. However, feedback for small systems like micro/nanoactuators is strongly limited by the difficulty to integrate the sensor: high bandwidth and enough accurate sensors are bulky, difficult to fabricate and very expensive (interferometers, triangulation optical sensors, cameramicroscopes measurement systems, etc.) [3]. Therefore, openloop control techniques - also called feedforward - have been used as an alternative. Although feedforward control can not reject the disturbance effects, this approach is well suited for micro/nanopositioning because the objects to be positioned have negligible masses and then the effect of the load is insignificant.

Feedforward consists in putting in cascade with the prosess a compensator resulting in an overall linearized system. Two kinds of compensator exist for piezoelectric actuators: compensator with charge control, and compensator with voltage control. Charge control based compensator [4] [5] [6] is based on an electrical circuit (charge integrator) that provides the input control (charge) to the piezoelectric actuator. Voltage control based compensator consists to accurately model the hysteresis and to put in cascade with the process the corresponding inverse model. Relative to charge control, voltage control does not require an additional electrical circuit and is of interest in a control theory (synthesis and analysis) point of view. For piezoelectric actuators, there exist two approaches of modeling and control used in voltage control: the Preisach [7] [8] [9] and the Prandtl-Ishlinskii approaches [3] [10] [11] [12]. In both, a complex hysteresis is modeled by the sum of many elementary hysteresis called hysterons. The compensator, i.e. the hysteresis inverse model, is afterwards computed using the identified model. On the one hand, the two approaches can be very accurate subject to the use of a high number of elementary hysteresis. However, the compensator complexity 
and implementation are compromised. On the other hand, a low number of elementary hysteresis compromises the accuracy. Finally, the additional computation of the inverse model is an additional constraint for these approaches.

In this paper, we propose a new approach to compensate the hysteresis of piezoelectric actuators. Based on the multiplicative-inverse structure, the proposed compensator scheme is adapted to hysteresis that is expressed by the BoucWen model. The advantage is that no more computation is required for the compensator. In fact, the same direct model is used in the compensator. Furthermore, as the Bouc-Wen model is based on a set of equations, the approach is very adapted in a control theory point of view. Therefore, the proposed approach presents an interest in term of simplicity of computation and implementation.

Section-2 is dedicated to the Bouc-Wen model of hysteresis in piezoelectric actuators. In section-3, we present the proposed compensation technique. Finally, experimental results and validation end the paper.

\section{BOUC-Wen HYSTERESIS MODELING}

\section{A. Remind of the Bouc-Wen model}

The Bouc model of hysteresis [13] - further modified by Wen [14] - was initially used for nonlinear vibrational mechanics. The Bouc-Wen model has an interesting simplicity and is able to represent a large class of hysteresis. It is based on a state variable $h$. In this model, the relationship between a mechanical excitation $F$ and the state $h$ is given by the following differential equation:

$$
\frac{d h}{d t}=A_{b w} \frac{d F}{d t}-B_{b w}\left|\frac{d F}{d t}\right| h|h|^{n-1}-\Gamma_{b w} \frac{d F}{d t}|h|^{n}
$$

where $A_{b w}$ controls the restoring force amplitude, $B_{b w}$ and $\Gamma_{b w}$ control the shape of the hysteresis loop and $n$ controls the smoothness of the transition from elastic to plastic response. Because of the elasticity of several piezoelectric actuators and especially cantilevered structure actuators, it is admitted that $n=1$ [15]. Replacing the mechanical input $F$ in (Eq 1) with the applied electrical voltage $U$, the static Bouc-Wen model adapted to piezoelectric actuators is described as follows [16]:

$$
\left\{\begin{array}{l}
y(t)=d_{p} U(t)-h(t), \quad y\left(t_{0}\right)=y_{0} \\
\frac{d h}{d t}=A_{b w} \frac{d U}{d t}-B_{b w}\left|\frac{d U}{d t}\right| h-\Gamma_{b w} \frac{d U}{d t}|h|, \quad h\left(t_{0}\right)=h_{0}
\end{array}\right.
$$

where $y$ is the displacement output and $U$ is the input control. Parameter $d_{p}$ represents the piezoelectric coefficient and is strictly positive. Many works reported on the identification procedure of these coefficients, example in [15] [18].

\section{B. Reduced expression of the model}

If we write $h=H(U)$, where $H(U)$ is a nonlinear operator characterized by the second equation of (Eq 2), the expression of the Bouc-Wen model is reduced as follows:

$$
y=d_{p} U-H(U)
$$

The previous reduced expression will be used for the compensator design in the next section. We remind that the BoucWen model is simple for implementation in an automatic point of view. Actually, this model can be easily transcribed into a block diagram ready-made for a Simulink implementation (Fig. 1).

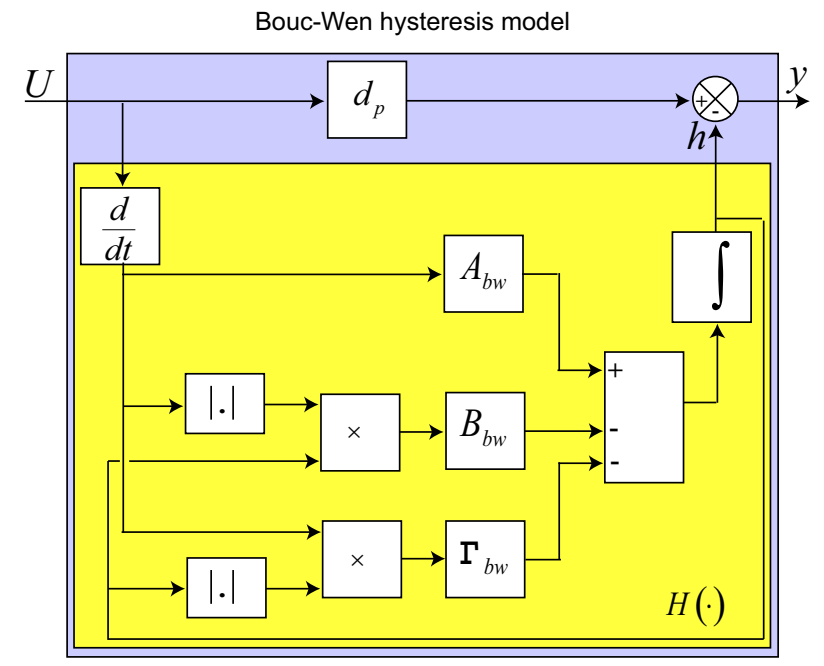

Fig. 1. Block diagram of the hysteresis model.

\section{A NEW APPROACH TO COMPENSATE THE HYSTERESIS}

A hysteresis compensator has the reference $y_{r}$ as input and the control signal $U$ as output (Fig. 2-a). The compensator can be seen as an inverse operation of the direct model, the objective being to have $y=y_{r}$. In existing works, the computation of the compensator is an additional work because its parameters have to be likewise computed. In our approach, this additional work is avoided.

Consider the direct model in (Eq 3), and let us extract the value of $U$ that meets the reference $y_{r}$, by only using the linear term $d_{p} U$. We obtain:

$$
U=\frac{1}{d_{p}}\left(y_{r}+H(U)\right)
$$

This expression will be used as the compensator which has an input $y_{r}$, an output $U$ and a nonlinear feedback $H(U)$. As we can see, the compensator does not require any inversion except for the real $d_{p}$ which is strictly positive. Furthermore, no additional computation is required because $d_{p}$ and $H(U)$ are already known during the modeling and identification. Finally, the compensator is simple in an implementation point of view because so is the Bouc-Wen model. The (reduced) block diagram of the compensator given by (Eq 4) is pictured in Fig. 2-b. It shows that the compensator has an inverse multiplicative structure. The above Bouc-Wen model is static, so the proposed approach is used to compensate static hysteresis. To consider a dynamic hysteresis, the static one can be combined with a linear dynamic model such as recommended in [2] [3] [8]. 

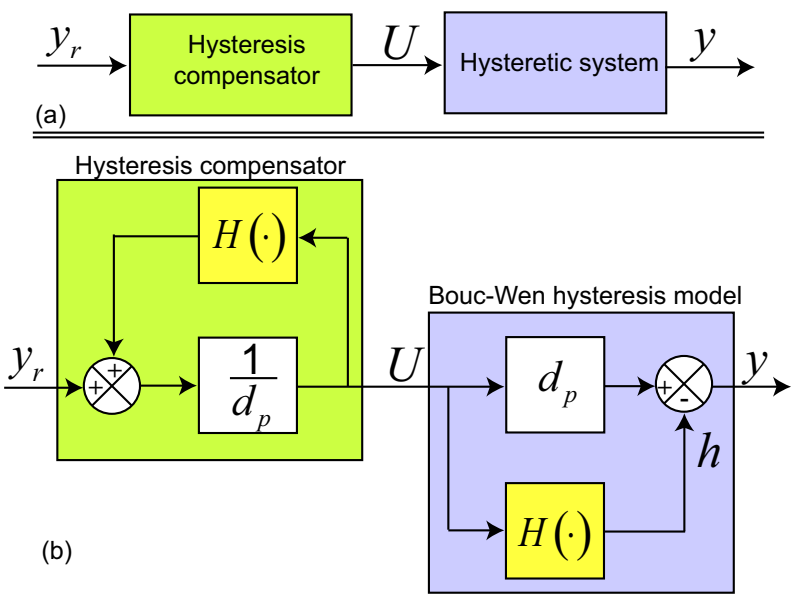

Fig. 2. Hysteresis compensation. (a): general principle. (b) the proposed structure.

\section{EXPERIMENTAL RESULTS}

The piezoelectric actuator used in this study is a unimorph cantilever with rectangular cross-section. Such an actuator is often used to develop microgrippers, walker microrobots, etc. [19]. A unimorph cantilever is made up of one piezoelectric layer (PZT) and one passive layer (Nickel). When applying a voltage to the piezolayer, it expands/contracts. As the piezolayer and the passive layer are glued themselves, they result a bending of the unimorph cantilever (Fig. 3-a). The setup, presented in Fig. 3-b, is based on:

- a unimorph with total sizes $15 \mathrm{~mm} \times 2 \mathrm{~mm} \times 0.3 \mathrm{~mm}$, where $0.2 \mathrm{~mm}$ and $0.1 \mathrm{~mm}$ are the thickness of the PZT and of the Nickel respectively,

- a computer and a dSPACE-board for generating the control signal $U$ and the reference $y_{r}$,

- a high-voltage amplifier,

- and an optical sensor used to report the bending $y$ of the actuator.

\section{A. Identification}

In this section, we identify the parameters of the BoucWen model (Eq 2). First by using a nonlinear filter system identification method [15], we provide approximated values of $A_{b w}, B_{b w}$ and $\Gamma_{b w}$. Afterwards, the identified parameters are validated by comparing the hysteresis curves of the model and of the actuator. During this step, the parameters can be manually refined if required. The hysteresis curves are obtained by applying a sine signal $U$ with a maximal amplitude of $80[\mathrm{~V}]$ to the actuator (resp. to the model) and by plotting the output versus this input. The maximal voltage amplitude corresponds to the maximal range of use. As the model is static, the frequency is chosen to be low in order to avoid the phase lag corresponding to the dynamics, and not too low to avoid the influence of the creep on the hysteresis curve [3]. A preliminary characterization of the used piezoelectric actuator shows that the effect of the creep on the hysteresis curve can be seen with a frequency lower than $0.01 \mathrm{~Hz}$, while

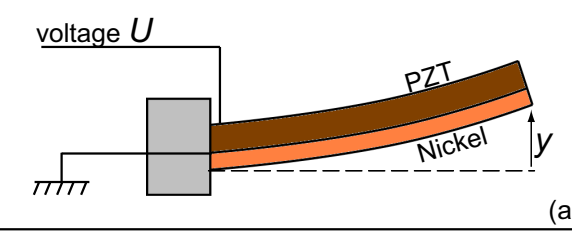

(a)

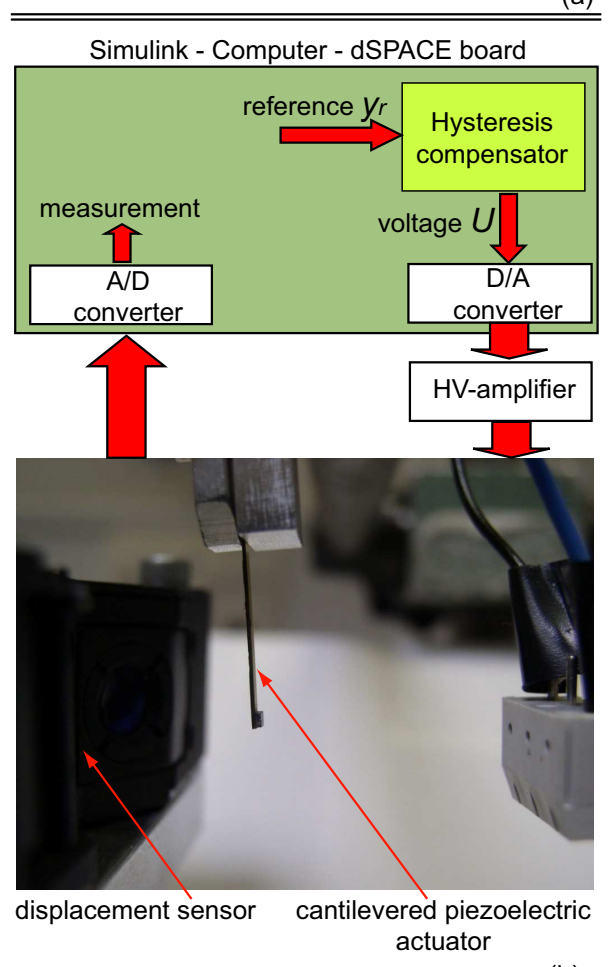

(b)

Fig. 3. Photography of the piezoelectric actuator.

the phase-lag due to the dynamics with a frequency higher than $5 \mathrm{~Hz}$. Therefore, we choose $0.1 \mathrm{~Hz}$ for the parameters identification and model validation. We obtain: $d_{p}=1.6\left[\frac{\mu m}{V}\right]$, $A_{b w}=0.9\left[\frac{\mu m}{V}\right]$ and $B_{b w}=\Gamma_{b w}=0.008\left[V^{-1}\right]$.

When plotting the characteristic $(U, y)$, we obtain the Fig. 4. It shows that the model simulation curve coincide with that of the experimental result. Therefore, the identified model well captures the hysteresis of the actuator.

\section{B. Hysteresis compensation}

Using the identified model, the structure of the compensator as in Fig. 2 and the detail of $H(\cdot)$ as in Fig. 1, we implemented the hysteresis compensator in the Simulink software.

The experiment consists in applying a sine input reference $y_{r}$ with an amplitude of $80[\mu \mathrm{m}]$ and a frequency of $0.1 \mathrm{~Hz}$. The result was compared with that of without compensator. It is shown that the initial hysteresis of nearly $56 \%$ (without compensator) has been completely removed when using the proposed compensator (Fig. 5-a) and the gain of the linearized overall system is equal to one. Fig. 5-b pictures the tracking performances. It shows that when using the hysteresis compensator, the output well tracks the input, while without compensator the tracking is lost. Indeed, the phase-lag between the output and the input is nearly $3^{\circ}$ with compensator while it 


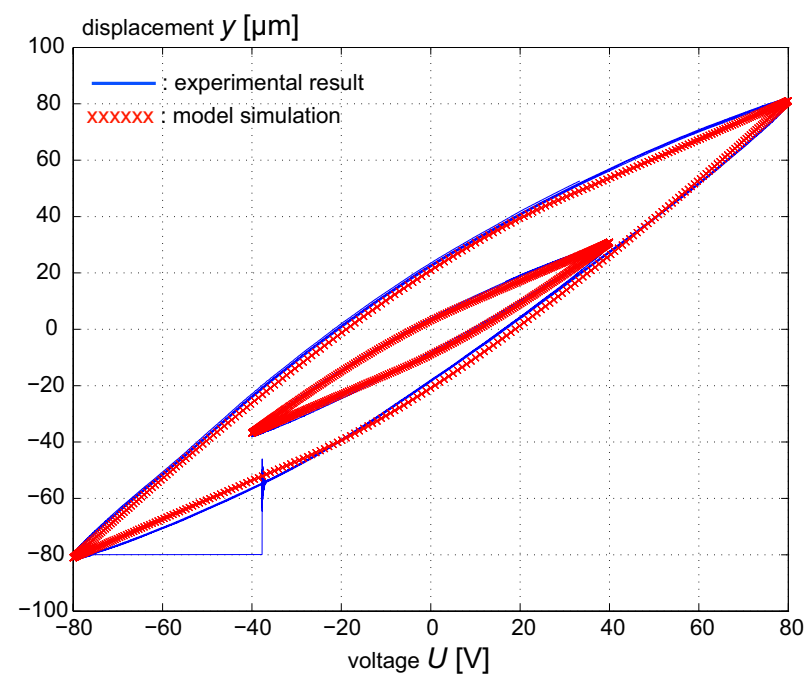

Fig. 4. The hysteresis of the piezoelectric actuator.

was $16^{\circ}$ without. Because the considered hysteresis modeling and compensation is static, the tracking performances are valuable for frequency between $0.01 \mathrm{~Hz}$ and $5 \mathrm{~Hz}$ as already explained above.
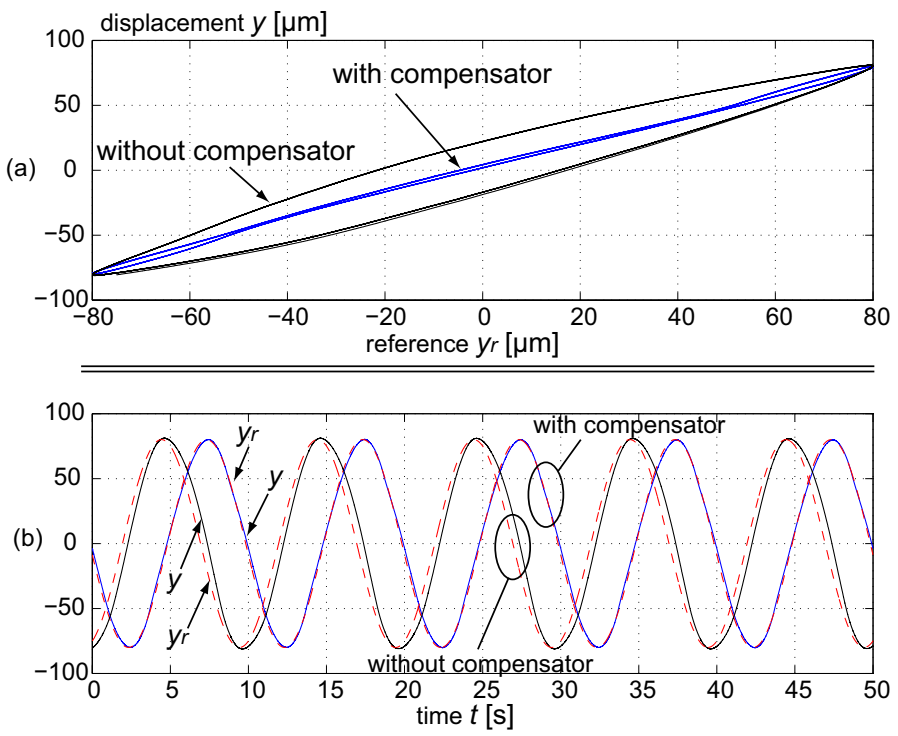

Fig. 5. Hysteresis with and without compensation: (a) input-output characteristics. (b) tracking characteristics.

\section{CONCLUSION}

We present a new approach to compensate the hysteresis nonlinearity in smart materials, especially piezoelectric, based actuators. The proposed approach is based on the Bouc-Wen modeling and the inverse multiplicative structure. Its main advantage relative to existing approaches is the simplicity of computation and implementation. Indeed, while no operator or model inversion is required, the compensator is available as soon as the direct model is identified. No additional computation is therefore required. The proposed approach is very useful for the accuracy improvement in actuators based on smart material, and where a simple compensator is demanded.

\section{ACKNOWLEDGMENT}

This work is supported by the Conseil Général du Doubs.

\section{REFERENCES}

[1] K. K. Leang and S. Devasia, "Hysteresis, creep, and vibration compensation", IFAC Conference on Mechatronic Systems, pp.283-289, 2002.

[2] M. Rakotondrabe, Y. Haddab and P. Lutz, "Quadrilateral modeling and robust control of a nonlinear piezoelectric cantilever", IEEE Transactions on Control Systems Technology, Vol.17, Issue 3, pp.528-539, May 2009.

[3] M. Rakotondrabe, C. Clévy and P. Lutz, "Complete open loop control of hysteretic, creepd and oscillating piezoelectric cantilevers", IEEE Transactions on Automation Science and Engineering, DOI 10.1109/TASE.2009.2028617.

[4] J.A Main, E. Garcia and D.V Newton, "Precision position control of piezoelectric actuators using charge feedback", J. Guid. Control Dyn., vol. 18, pp.1068-73, 1995.

[5] J. Agnus and N. Chaillet, "Dispositif de commande d'un actionneur pizolectrique et scanner muni de ceux-ci", INPI Patent, $n^{o}$ FR03000532, 2003.

[6] G. M. Clayton, S. Tien, A. J. Fleming, S. O. R. Moheimani, S. Devasia, "Inverse-feedforward of charge-controlled piezopositioners ", Mechatronics, Volume 18, Number 5-6, page 273-281 - June 2008.

[7] D. Hughes and J. T. Wen, "Preisach modeling of piezoceramic and shape memory alloy hysteresis", Smart Materials and Structures, Vol.4, pp.287399, 1997.

[8] D. Croft, G. Shed and S. Devasia, "Creep, hysteresis and vibration compensation for piezoactuators: atomic force microscopy application", ASME Journal of Dynamic Systems, Measurement and Control, 2001.

[9] A. Dubra and J. Massa and C.1 Paterson, "Preisach classical and nonlinear modeling of hysteresis in piezoceramic deformable mirrors", Optics Express, Vol.13, $N^{\circ} 22$, pp.9062-9070, 2005.

[10] K. Kuhnen and H. Janocha, "Inverse feedforwrad controller for complex hysteretic nonlinearities in smart-materials systems", Control of Intelligent System, Vol.29, $N^{\circ} 3,2001$.

[11] W. T. Ang, P. K. Kholsa and C. N. Riviere, "Feedforward controller with inverse rate-dependent model for piezoelectric actuators in trajectory-tracking applications", IEEE/ASME Transactions on Mechatronics (Tmech), Vol.12(2), pp.134-142, April 2007.

[12] B. Mokaberi and A. A. G. Requicha, "Compensation of scanner creep and hysteresis for AFM nanomanipulation", IEEE Transactions on Automation Science and Engineering, Vol.5, $N^{o} 2$, pp.197-208, 2008.

[13] R. Bouc, "Forced vibration of mechanical systems with hysteresis", Conference on Nonlinear Oscillation, Prague, 1967.

[14] Y. K. Wen, "Method for random vibration of hysteresis systems", Journal of the Engineering Mechanics Division, Vol. 102(2), pp.249-263, March/April 1976.

[15] M. Jouaneh and H. Tian, "Accuracy enhancement of a piezoelectric actuators with hysteresis", ASME Japan/USA Symp. Flexible Automation, pp.631-637, 1992.

[16] T. S. Low and W. Guo, "Modeling of a three-layer piezoelectric bimorph beam with hysteresis", Journal Microelectromechanical Systems, Vo.4(4), pp.230-237, December 1995.

[17] C. W. Wong, Y. Q. Ni and J. M. Ko, "Steady state oscillation of hysteretic differential model. I - response analysis, II - performance analysis", Journal Engineering Mechanics, 120, 1994.

[18] C. W. Wong, Y. Q. Ni and J. M. Ko, "Steady state oscillation of hysteretic differential model. I - response analysis, II - performance analysis", Journal Engineering Mechanics, 120, 1994.

[19] S.K. Naha and Z.W. Zhong, "A microgripper using piezoelectric actuation for micro-object manipulation", Sensors and Actuators A: Physical, Vol.133(1), January 2007. 